\title{
Lagrange Multiplying Method based Calculation of Material Flaw Thickness Exploiting Ultrasonic Multipath Detection
}

\author{
X.Z. Shen, S.J. Chen \\ Electrical and Automatic School \\ Shanghai Institute of Technology \\ Shanghai, China, 201418
}

\begin{abstract}
A signal model based on the multipath higher order reflections of the flaw is reviewed to detect the sized flaw by ultrasound nondestructive testing, and the different paths are pre-defined and identified to form the corresponding equations. Three paths are studied, DRP, MP-C and MP-W. DRP and MP-C are utilized to measure the flaw from the top, and MP-W is from its bottom. Ultrasonic imaging is formed and synthesized by all the identified paths and all the tests to make the thickness of flaw be revealed. The thickness of the flaw is turned to two optimization problems based on different path profiles by only one pitch-catch measurement, and the problem is estimated based on Lagrange multiplying method. Simulations and experimentations demonstrate that the flaw sizing can be calculated utilizing the time-of-arrivals of the multipath signals, even with only one pitch-catch measurement.
\end{abstract}

Keywords-ultrasonic nondestructive testing; multipath; sizing; flaw; lagrange multiplying method

\section{INTRODUCTION}

Sizing of material flaws is an important problem in ultrasonic nondestructive evaluation (NDE)[1]. One of the direct methods of flaw sizing is B-scan imaging from at least two different profiles, and transducer arrays are practical. However, when the space of installation of transducers is limited, it is hard to install enough sensors and the size is thus difficult to be observed. Therefore, it is necessary to develop a method to detect the Thickness with small number of transducers, or even with only one pair of transducers.

In this paper, we propose a method of sizing an isolated flaw using pitch-catch measurements. The multipahs are reviewed to show that the fusion of physical and virtual sensor data makes sizing the flaw possible[2][3]. Different from[2][3], an optimal model is given in this paper, which is solved by Lagrange multiplying method.

\section{Multipath Signal Model AND SCENARIOS}

\section{A. Multipath Signal}

Consider an ultrasonic pitch-catch measurement system. We model the measured ultrasound signal [2][3] at the receiving transducer, $r_{m n}(t)$ as follows,

$$
r_{m n}(t)=\sum_{i} \alpha_{i, m n} s\left(t-\tau_{i, m n}\right)
$$

wheres $(t)$ is the ultrasonic pulse-echo wavelet, $\alpha_{i, m n}$ and $\tau_{i, m n}$ are respectively $i$-th path reflectivity and delay time of $m$-th transmitter and $n$-th receiver.See Figure 1 .

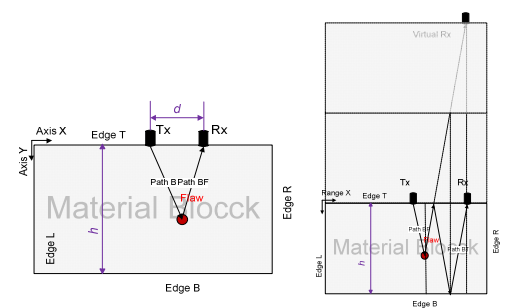

(a)DRP(b) MP-1

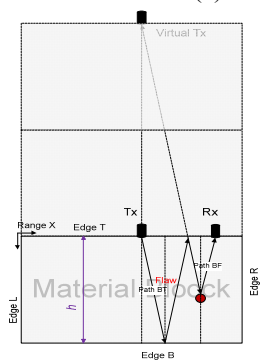

(c) MP-2

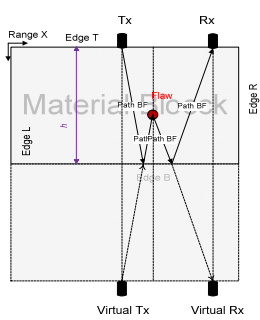

(d) MP-W
FIGURE I. DIRECT REFLECTION AND MULTIPATH SCENARIOS IN A PITCH-CATCH MEASUREMENT.

\section{B. Multipath Delays and Equations}

Consider a region of interest, which is a two-dimensional cross-section under the linear array, and a receiving mode backprojection beamforming algorithm is utilized to construct the image of the cross-section in interior material [3]. A sized flaw is assumed to be circle-like convex with its center at the position $p_{\mathrm{f}}=\left(x_{\mathrm{f}}, y_{\mathrm{f}}, z_{\mathrm{f}}\right)^{\mathrm{T}}$ and its diameter $d$, and its top point at $p_{\mathrm{ft}}=\left(x_{\mathrm{ft}}, y_{\mathrm{ft}}-\frac{d}{2}, z_{\mathrm{ft}}\right)^{\mathrm{T}}$, and its bottom point at $p_{\mathrm{fb}}=$ $\left(x_{\mathrm{fb}}, y_{\mathrm{fb}}+\frac{d}{2}, z_{\mathrm{fb}}\right)^{\mathrm{T}}$. The $m$ th transmitting transducer is assumed to be located at $p_{t, m}=\left(x_{t, m}, y_{t, m}, z_{t, m}\right)^{\mathrm{T}}$, and the $n$th 
receive transducer located at $p_{r, n}=\left(x_{r, n}, y_{r, n}, z_{r, n}\right)^{\mathrm{T}}$. In general, we set $z_{\mathrm{f}}=z_{\mathrm{ft}}=z_{\mathrm{fb}}=z_{t, m}=z_{r, n}=0$.

The signal corresponding to DRP recorded at the $n$th receive transducer and $m$-th transmitter is given by

$$
r_{0, m n}(t)=\alpha_{0, m n} s\left(t-\tau_{0, m n}\right)
$$

where $\alpha_{0, m n}$ is the DRP reflectivity of the flaw, and $\tau_{0, m n}$ denotes the delay of the wavelet to travel for a round-like trip of DRP. Assuming a homogeneous material with constant ultrasonic propagation speed of $v$ in the material, the time delay of DRP corresponding to $p_{\mathrm{ft}}$ can be calculated as

$$
\tau_{0, m n}=\left(\left\|p_{t, m}-p_{f t}\right\|+\left\|p_{r, n}-p_{f t}\right\|\right) / v
$$

Denote $h$ as the thickness of the metallic object. The virtual transducer positions are respectively located at $p_{t 1, m}=$ $p_{t, m}, p_{r 1, n}=\left(x_{r, n}, y_{r, n}-2 h, z_{r, n}\right) \quad$ for $\quad$ MP-1, $\quad p_{t 2, m}=$ $\left(x_{t, m}, y_{t, m}-2 h, z_{t, m}\right), p_{r 2, n}=p_{r, n}$ for MP-2, and $p_{t W, m}=$ $\left(x_{t, m}, 2 h-y_{t, m}, z_{t, m}\right), p_{r W, n}=\left(x_{r, n}, 2 h-y_{r, n}, z_{r, n}\right) \quad$ for MP-W. The time delays of MP-1, MP-2 and MP-W corresponding to a sized target can be respectively calculated as

$$
\mathrm{MP}-1: \tau_{1, m n}=\left(\left\|p_{t 1, m}-p_{f t}\right\|+\left\|p_{r 1, n}-p_{f t}\right\|\right) / v
$$

MP-2:

$\tau_{2, m n}=\left(\left\|p_{t 2, m}-p_{f t}\right\|+\left\|p_{r 2, n}-p_{f t}\right\|\right) / v$

MP-W:

$\tau_{W, m n}=\left(\left\|p_{t W, m}-p_{f b}\right\|+\left\|p_{r W, n}-p_{f b}\right\|\right) / v$

The ranges of multipaths, MP-1 and MP-2, are so close that they may be overlapped partly or completely. So we only can detect the combined overlapped version of MP-1 and MP-2, or the stronger one of the two if they are not overlapped, and it is denoted as MP-C. The TOA range trajectory of MP-C can be found as,

$$
\tau_{C, m n}=\left\{\begin{array}{c}
\frac{\left\|p_{t 1, m}-p_{\mathrm{ft}}\right\|+\left\|p_{r 1, n}-p_{\mathrm{ft}}\right\|}{v}, \\
\left|x_{f t}-x_{t m}\right|<\left|x_{f t}-x_{r n}\right| \\
\frac{\left\|p_{t 2, m}-p_{\mathrm{ft}}\right\|+\left\|p_{r 2, n}-p_{\mathrm{ft}}\right\|}{v}, \\
\left|x_{f t}-x_{t m}\right| \geq\left|x_{f t}-x_{r n}\right|
\end{array}\right.
$$

The top point, $p_{\mathrm{ft}}$, also locates at the locus of DRP, and then the implicit function $\tau_{C, m n}$ can be obtained only with respect to $x_{\mathrm{ft}}$.

\section{Multipath Equations}

If we have detected the above TOAs, $\tau_{0, m n}, \tau_{\mathrm{C}, m n}$ and $\tau_{\mathrm{W}, m n}$, the corresponding equations are respectively described as

DRP: $\quad \sqrt{\left(x_{t m}-x_{f t}\right)^{2}+\left(y_{t m}-y_{f t}\right)^{2}}+$
$\sqrt{\left(x_{r n}-x_{f t}\right)^{2}+\left(y_{r n}-y_{f t}\right)^{2}}=v \tau_{0, m n}$

MP-C:

$$
\begin{aligned}
& \sqrt{\left(x_{t m}-x_{f t}\right)^{2}+\left(y_{t m}-y_{f t}\right)^{2}} \\
& +\sqrt{\left(x_{r n}-x_{f t}\right)^{2}+\left(y_{r n}-2 h-y_{f t}\right)^{2}} \\
& =v \tau_{\mathrm{C}, m n},\left|x_{f t}-x_{t m}\right|<\left|x_{f t}-x_{r n}\right| \\
& \sqrt{\left(x_{t m}-x_{f t}\right)^{2}+\left(y_{t m}-2 h-y_{f t}\right)^{2}} \\
& +\sqrt{\left(x_{r n}-x_{f t}\right)^{2}+\left(y_{r n}-y_{f t}\right)^{2}} \\
& =v \tau_{\mathrm{C}, m n},\left|x_{f t}-x_{t m}\right| \\
& \geq\left|x_{f t}-x_{r n}\right|
\end{aligned}
$$

MP-W:

$$
\begin{gathered}
\sqrt{\left(x_{t m}-x_{f b}\right)^{2}+\left(2 h+y_{t, m}-y_{f b}\right)^{2}} \\
+\sqrt{\left(x_{r n}-x_{f b}\right)^{2}+\left(2 h+y_{r n}-y_{f b}\right)^{2}}=v \tau_{\mathrm{W}, m n}
\end{gathered}
$$

These equations form 3 ellipses with focus points at the points of transducers. And the equation of DRP in (8) can be also expressed as

$$
\begin{aligned}
& \frac{\left(x_{f t}-\frac{x_{t m}+x_{r n}}{2}\right)^{2}}{\frac{\left(x_{t m}-x_{r n}\right)^{2}+\left(y_{t m}-y_{r n}\right)^{2}}{4}} \\
& +\frac{\left(y_{f t}-\frac{y_{t m}+y_{r n}}{2}\right)^{2}}{\left(\frac{v \tau_{0, m n}}{2}\right)^{2}-\frac{\left(x_{t m}-x_{r n}\right)^{2}+\left(y_{t m}-y_{r n}\right)^{2}}{4}}=1
\end{aligned}
$$

\section{OptimizATION Model AND ItS SOlutions OF FlAW SIZING}

We redraw all the multipaths in Figure 2, where DRP with blue locus and ways, MP-C with orange locus and ways, MP-W with red locus and ways, and the flaw with red circle. The multipaths, DRP and MP-C, go through the top point of the 
flaw, and the multipath, MP-W, goes through the bottom point of flaw. The size of the flaw can thus be modeled as the following optimal problem,

$$
\begin{aligned}
& d=\min \left\|p_{f t}-p_{f b}\right\| \\
& =\min \sqrt{\left(x_{f t}-x_{f b}\right)^{2}+\left(y_{f t}-y_{f b}\right)^{2}}
\end{aligned}
$$

Subject to

$$
\begin{gathered}
\begin{aligned}
f_{0, m n}=\sqrt{\left(x_{t m}-\right.} & \left.x_{f t}\right)^{2}+\left(y_{t m}-y_{f t}\right)^{2} \\
& +\sqrt{\left(x_{r n}-x_{f t}\right)^{2}+\left(y_{r n}-y_{f t}\right)^{2}} \\
& -v \tau_{0, m n}=0
\end{aligned} \\
f_{\mathrm{W}, \mathrm{mn}}=\sqrt{\left(x_{t m}-x_{f b}\right)^{2}+\left(2 h+y_{t, m}-y_{f b}\right)^{2}} \\
\quad+\sqrt{\left(x_{r n}-x_{f b}\right)^{2}+\left(2 h+y_{r n}-y_{f b}\right)^{2}} \\
-v \tau_{W, m n}=0
\end{gathered}
$$

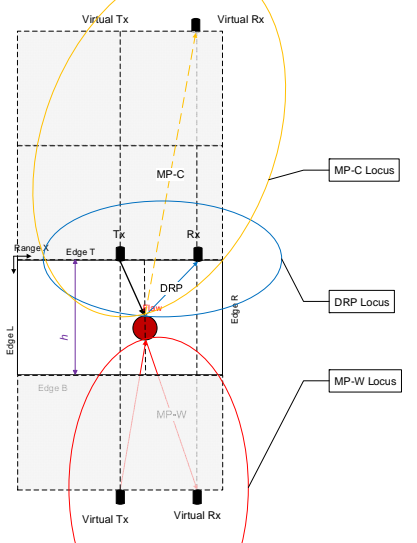

FIGURE II. DETECTION OF FLAW SIZE.
We here apply Lagrange multiplying method [6] to solve the optimization problem in(12). The segmented constrained equal equations can be seen as two problems. Thus, we construct two Lagrange functions as follows,

$$
\begin{aligned}
L_{1}=\sqrt{\left(x_{f t}-x_{f b}\right)^{2}+\left(y_{f t}-y_{f b}\right)^{2}} \\
+\sum_{m, n}\left(\lambda_{0, m n} f_{0, m n}+\lambda_{W, m n} f_{\mathrm{W}, \mathrm{mn}}\right. \\
\left.+\lambda_{1, m n} f_{\mathrm{C} 1, m n}\right)
\end{aligned}
$$

and,

$$
\begin{aligned}
L_{2}=\sqrt{\left(x_{f t}-x_{f b}\right)^{2}+\left(y_{f t}-y_{f b}\right)^{2}} & \\
& +\sum_{m, n}\left(\lambda_{0, m n} f_{0, m n}+\lambda_{W, m n} f_{\mathrm{W}, \mathrm{mn}}\right. \\
& \left.+\lambda_{1, m n} f_{\mathrm{C} 2, m n}\right)
\end{aligned}
$$

As to the symmetry of MP-1 and MP-2 with respect to the center line of the transducers, the solution of the problem in (13) is theoretically the same as in (14). So we only consider one of them, such as the one in (13). However, we solve both problems and get two minimum values, and take the smaller value as the thickness of the flaw.

Now, we solve the problem in (13), and the procedure is also applied to(14). Set $x=\left[x_{f t}, y_{f t}, x_{f b}, y_{f b}, \lambda_{0, m n}, \lambda_{W, m n}, \lambda_{1, m n}\right]^{\mathrm{T}}$. The $k$-step update version of $x$ is as follows,

$$
x_{k+1}=x_{k}-\left.\mu \frac{\mathrm{d} L}{\mathrm{~d} x}\right|_{x=x_{k}}
$$

Here, $\mu$ is the step-size, which can be decided by the optimization step-size, $\mu_{0}=\operatorname{argmin}_{\mu} L\left(x_{k+1}\right) \cdot \frac{\mathrm{d} L}{\mathrm{~d} x}$ isthe gradient of Lagrange function, and here $L$ can be either $L_{1}$ or $L_{2}$. When $\left|\frac{\mathrm{d} L}{\mathrm{~d} x}\right|_{x=x_{k}} \mid$ is less than one given terminal constants, such as $10^{-5}$ or the iterative number of steps is up to a given number, we terminate the updated iterative operation. After we get the minimum value of $x$, we can calculate the value of $d$ in(12), and thus we get two values, $d_{1}$ and $d_{2}$ from the problems in (13) and. Then we take $d=\min \left(d_{1}, d_{2}\right)$.

\section{EXPERIMENTAL RESULTS}

\section{A. Experiment Setup}

An aluminum block, alloy type 6061 is used to study the ultrasound flaw sizing. A side through hole $(\mathrm{STH})$ of $\Phi 2 \mathrm{~mm}$ diameter is drilled into the material from side to simulate a sized 
specular flaw.Seven transducers are located on the top surface of the material and their x-positions related to the flaw center are aligned equally from $-30 \mathrm{~mm}$ to $30 \mathrm{~mm}$ with $10 \mathrm{~mm}$ increment. 42 set of signals are sampled at $40 \mathrm{MHz}$ sampling rate. The DRP of the flaw appear around $16 \mu$ s, whereas MP-W is located around $30.5 \mu \mathrm{s}$. MP-C is located around $39.3 \mu$ s.All the waveform envelopes show clear DRP, approximately between $16.0 \mu \mathrm{s}$ and $18.5 \mu \mathrm{s}$, and $1^{\text {st }}$-order bottom reflection, approximately between $23.5 \mu$ s and $25.1 \mu \mathrm{s}$.

\section{B. Ultrasonic Imaging}

We have 42 set of data, and each set can be used as one path in (13) or to give overdetermined constraint equation. However, we here don't discuss how to solve the problem of so much constraint equations, but to discuss the ability of multipaths. We apply ultrasound imaging to show the possible solution of the flaw. Figure 3 shows the imaging results using different paths [3]. Figure 3a shows the image generated using DRP. The flaw top is identified. MP-W yields the bottom view of the flaw in Figure 3b. Figure 3c shows the top view obtained from MP-C. These images represent reflections at different profiles, and image fusion can be applied[3], shown in Figure 3d. The size of the hole is revealed by the fusion result, which is otherwise unavailable if only DRP observations are alone exploited. By observation, the thickness of the flaw is around $3-5 \mathrm{~mm}$.

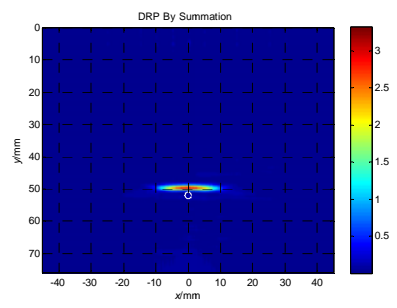

(a)DRP

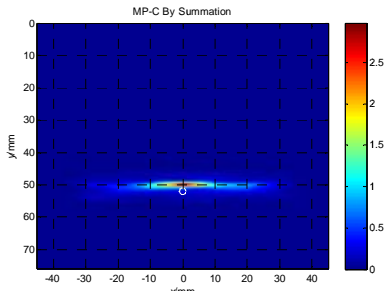

(c) MP-C
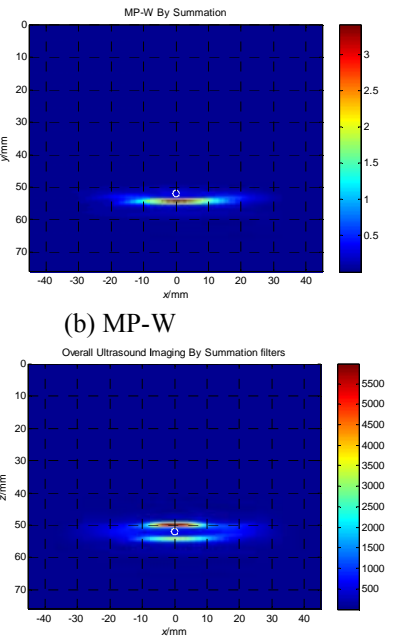

(d) Overall synthesis (b) MP-W

FIGURE III. ULTRASOUND IMAGING OF A HOLE VIA MULTIPATH EXPLOITATION.

\section{Optimal Algorithm of Thickness}

To show the ability of extended data due to the mulitpaths, we show here only one pitch-catch measurement can be applied to solve the size of the flaw. Figure4 shows the estimated thickness of the STH by the proposed method, where Figure4a shows the solutions of the thickness, the blue dotted line is solved by the optimal problem in (13) and the red dotted line is solved by the problem in. We can see that both solutions in problems (13) and are nearly the same in the sense that both the average thicknesss of the flaw is $3.3995 \mathrm{~mm}$. We take the minimum value of the solutions by (13) and as the estimated thickness of the STH, and depicted in Figure $4 \mathrm{~b}$, whose mean value is $3.19 \mathrm{~mm}$.

\section{SUMMARY}

Ultrasounds along different paths have different profiles of the flaw. We identifythe direct reflection path using conventional techniques, and then estimate the delay times of MP-C and MP-W in their active region. Thus we identify the multipaths, MP-C and MP-W. By fusing the identified ultrasonic multipaths, we can intuitively observe the size of the flaw. The flaw top point is formed by DRP and MP-C, and the flaw bottom point is exploited by MP-W. Thus the size from top to bottom of the flaw is observed by the ultrasound imaging of the flaw. At last, we turned it to an optimal problem, which is an equality constraint optimal problem. Lagrange multiplying method is applied to solve the problem, and the solution represent the thickness of flaw. Experiments shows the method is valid, although it is greater than the machined simulated hole.
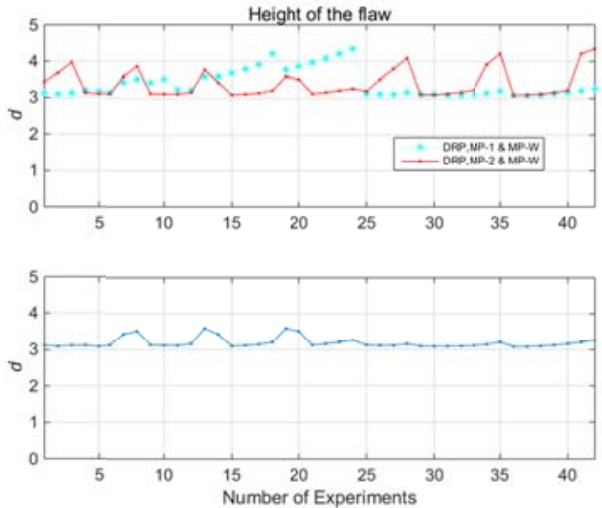

FIGURE IV. (a) ESTIMATED THICKNESS OF THE FLAW, THE BLUE DOTTED LINE BY THE OPTIMAL PROBLEM IN (17) AND THE RED DOTTED LINE BY THE PROBLEM IN (18); (b) THE MINIMUM VALUE OF THE SOLUTIONS.

\section{ACKNOWLEDGEMENTS}

This paper is supported by STCSM, No. 15ZR1440700.

\section{REFERENCES}

[1] L. W. Schmerr and S.-J. Song, Ultrasonic Nondestructive Evaluation Systems, Springer, 2007.

[2] Y. Zhang, X. Shen, R. Demirli and M. G. Amin, "Ultrasonic Flaw Imaging via Multipath Exploitation". Advances in Acoustics and Vibration. Vol. 2012 (2012), pp: 1-12.

[3] Xizhong Shen, Li Pan. Material Flaw Sizing By Ultrasonic Multipath Detection. Periodical of Advanced Materials Research Vols. 712-715, 2013, pp: 1067-1070.

[4] G. Alli and D. DiFlippo, "Beamforming for through-the-wall radar imaging," Chapter 3, in M. G. Amin (Ed.), Through the Wall Radar Imaging, CRC Press, 2010.

[5] Shen XZ, Yimin Z. et al. Ultrasound multipath background clutter mitigationBased on Subspace Analysis and projection.ICASSP 2012, March 25-30, 2012, Kyoto, Japan, pp: 2505-2508.

[6] Bertsekas D P, Nedic A, Ozdaglar A E. Convex Analysis and optimization. Athena Scientific Press, 2003. 